\title{
SUR LA DURÉE DE PRÉSENCE DES ENGRAIS PHOSPHATES DANS L'EAU ET LEUR ACTION SUR LE MILIEU
}

\author{
par J. MOREAU \\ Ecole Nationale Supérieure Agronomique \\ Université de Madagascar \\ Avec la collaboration technique de CAKPO Télesphore
}

\begin{abstract}
RESUME
Les essais ont èté réalisés à la Station piscicole de BOUAKE (Côte d'Ivoire).

Aux doses de 20 et $40 \mathrm{~kg} / \mathrm{ha}$ de superphosphate triple à $45 \%$ de $\mathrm{P}_{2} \mathrm{O}_{5}$ dans des étangs non empoissonnés, les phosphates demeurent respectivement 8 et 15 jours dissous dans l'eau; aux doses de 80 et $120 \mathrm{~kg} / \mathrm{ha}$, ils persistent un peu plus de 3 semaines. Parmi les modifications de milieux imputables à la présence des phosphates, figurent un relèvement du $\mathrm{PH}$ et du SBV et un déficit en fer par rapport aux étangs témoins. Cette dernière observation permet d'émettre des hypothèses sur le devenir des phosphates épandus dans l'étang de la Station de BOUAKE. Les essais sont comparés aux travaux analogues exécutés dans une station piscicole de MADAGASCAR.
\end{abstract}

\section{INTRODUCTION}

La fertilisation des étangs a été très tôt considérée comme un excellent moyen d'en augmenter la production piscicole; par rapport à l'alimentation, elle présente souvent l'avantage d'être plus èconomique et, de toute façon, elle nécessite des ẻpandages beaucoup moins fréquents que les quotidiennes dis- 
tributions de nourriture; de plus, elle ne fait pas courir le risque de maladies alimentaires et améliore même, dans certains cas, l'état sanitaire des étangs (HUET, 1970).

Depuis longtemps, les pisciculteurs de divers pays ont cherché à déterminer, de façon empirique, les doses optimales d'engrais à épandre et la périodicité de ces épandages. Leurs travaux ont donné lieu à de nombreuses publications (symposium de la FAO - ROME - 1966). Peu d'auteurs ont cherché à connaitre la durée de présence, en solution dans l'eau, des engrais et leur devenir après épandage. Les seules ètudes à citer sont celles de GOLTERMAN (1968), RABANAL (1968) et VINCKE (1969-70).

Ces données conditionnent, en fait, le rythme d'épandage à adopter, compte tenu de la dose choisie.

\section{CONDITIONS D'EXPERIENCES, MATERIELS ET METHODES}

Les travaux rapportés ci-après ont été menés à la Station piscicole de Bouaké (Côte d'lvoire) située à $300 \mathrm{~km}$ au Nord d'Abidjan, à $400 \mathrm{~m}$ d'altitude sur sol latéritique. Le climat est celui de la zone soudano-guinéenne, avec, en principe, une saison sèche et une saison humide, chacune longue d'environ 6 mois; en fait, il est très rare qu'il n'y ait pas au moins une pluie sur toute la durée d'un mois dit "sec".

Les doses suivantes de superphosphate calcique triple à $45 \%$ de $\mathrm{P}_{2} \mathrm{O}_{3}$ ont été épandues : $20 \mathrm{~kg} / \mathrm{ha}, 40 \mathrm{~kg} / \mathrm{ha}, 80 \mathrm{~kg} / \mathrm{ha}, 120 \mathrm{~kg} / \mathrm{ha}$. Chaque dose a fait l'objet de quatre répétitions en étang non empoisonné de $400 \mathrm{~m}^{2}$ et représente respectivement $9,18,36$ et $54 \mathrm{~kg}$ de $\mathrm{P}_{2} \mathrm{O}_{5} / \mathrm{ha}$.

Les engrais ont été déposés dans 2 paniers, en partie immergés sur la diagonale de l'étang opposée à celle qui relie le moine à l'arrivée d'eau.

Des prélèvements d'eau, en vue d'analyses ultérieures, ont été réalisés à $8 \mathrm{H}$ du matin, près du moine, la veille des épandages, le lendemain, le surlendemain, puis tous les 3 jours en moyenne jusqu'à ce qu'il n'y ait plus de phosphates dissous dans l'eau. résistivité.

Les mesures suivantes ont èté effectuées : température, $\mathrm{PH}, \mathrm{SBV}$,

Les éléments suivants ont été dosés par méthode colorimétrique à l'aide du comparateur Hellige.

\begin{tabular}{|c|c|c|}
\hline Elément & Quantité minin & male décelée \\
\hline $\begin{array}{l}\mathrm{P}_{2} \mathrm{O}_{5} \\
\mathrm{Fer} \\
\mathrm{NH}_{4} \\
\mathrm{NO}_{2} \\
\mathrm{NO}_{3}\end{array}$ & $\begin{array}{l}0,05 \\
0,05 \\
0,125 \\
0,002 \\
0,3\end{array}$ & $\begin{array}{l}\mathrm{mg} / \mathrm{l} \\
\mathrm{mg} / \mathrm{l} \\
\mathrm{mg} / \mathrm{l} \\
\mathrm{mg} / \mathrm{l} \\
\mathrm{mg} / \mathrm{l}\end{array}$ \\
\hline
\end{tabular}

En outre, la quantité d'oxygène dissous dans l'eau a été mesurée sur les prélèvements effectués les mêmes jours que précédemment, mais à $16 \mathrm{H}$. 


\section{RESULTATS}

$\mathrm{Si}$, d'une fois sur l'autre, les analyses d'eau, surtout au début des essais, ont quelquefois donné des résultats différents, la durée de présence des engrais phosphatés a été constante pour une même dose d'engrais entre les différentes répétitions. Les résultats sont résumés ci-dessous.

\begin{tabular}{|c|c|c|c|c|}
\hline DOSES & $\frac{20 \mathrm{~kg} / \mathrm{ha}}{8}$ & $\frac{40 \mathrm{~kg} / \mathrm{ha}}{17}$ & $\frac{80 \mathrm{~kg} / \mathrm{ha}}{23}$ & $\frac{120 \mathrm{~kg} / \mathrm{ha}}{26}$ \\
\hline $\begin{array}{c}\text { Durée de la présence } \\
\text { jans l'eau }\end{array}$ & $\begin{array}{c}26 \\
\text { jours }\end{array}$ & $\begin{array}{c}26 \\
\text { jours }\end{array}$ \\
\hline
\end{tabular}

L'évolution moyenne dans le temps de la quantité de $\mathrm{P}_{2} \mathrm{O}_{5}$ présente dans l'eau pour chaque quantité d'engrais épandu est reprise sur les figures 1 à 4 (graphique du bas, en trait plein). suivantes :

Les autres analyses d'eau nous ont permis de faire les observations

Le PH : L'augmentation (maximum observé 0,4) a été particulièrement sensible aux faibles doses d'engrais et un peu moins aux doses élevées, sans raison apparente (fig. 1 à 4). (Valeurs observées PH 7 à 8 ).

Le SBV : Ses variations ont suivi dans l'ensemble celles du PH (fig. 1 à 4); les valeurs observées au cours de l'expérience variaient de 1 à 1,5.

Le Fer : On observe, pendant la durée de présence des engrais dans l'eau, une diminution de la teneur en fer des étangs fertilisés par rapport aux témoins. Les moyennes des données concernant ces observations sont consignées par traitement sur les figures 1 à 4 (voir discussion). Les teneurs en fer observées ont varié de 0,1 à $0,5 \mathrm{mg} / \mathrm{l}$.

L'Aınmoniaque : A ${ }_{i x}$ doses élevées d'engrais (80 et $120 \mathrm{~kg} / \mathrm{ha}$ ), il existe également une diminution sensible de la concentration en Ammoniaque par rapport aux témoins (figures 3 et 4); ceci apparait moins aux doses inférieures, ainsi qu'en témoigne le tableau I ci-dessous.

TABLEAU I

Déficit en Ammoniaque ( $\mathrm{mg} / \mathrm{litre})$

\begin{tabular}{|c|c|c|c|c|c|c|}
\hline Dose $^{\text {Jour }}$ & 1 & 2 & 4 & 7 & 10 & 14 \\
\hline $20 \mathrm{~kg}$ & $+0,05$ & $+0,035$ & 0 & 0,07 & 0,02 & 0,02 \\
\hline $40 \mathrm{~kg}$ & $+0,17$ & $+0,06$ & 0,2 & 0,07 & $+0,1$ & $+0,15$ \\
\hline
\end{tabular}

N.B. : Le signe + signifie qu'il y a excédent de $\mathrm{NH}_{4}$ par rapport au témoin.

Aux doses élevées, l'état sanitaire des ètangs semble donc amélioré. Les quantités d'Ammoniaque présentes dans l'eau variaient de 0,125 à $0,80 \mathrm{mg} / \mathrm{litre}$. 
Fig. 1 à 4

RESUME DES OBSERVATIONS FAITES A LA STATION PISCICOLE DE BOUAKE (RCI)

Fig. $1-20 \mathrm{~kg} / \mathrm{ha}$ de supertriple $=9 \mathrm{~kg} \mathrm{P}_{2} \mathrm{O}_{5} / \mathrm{ha}$ (chaque point représente une moyenne)
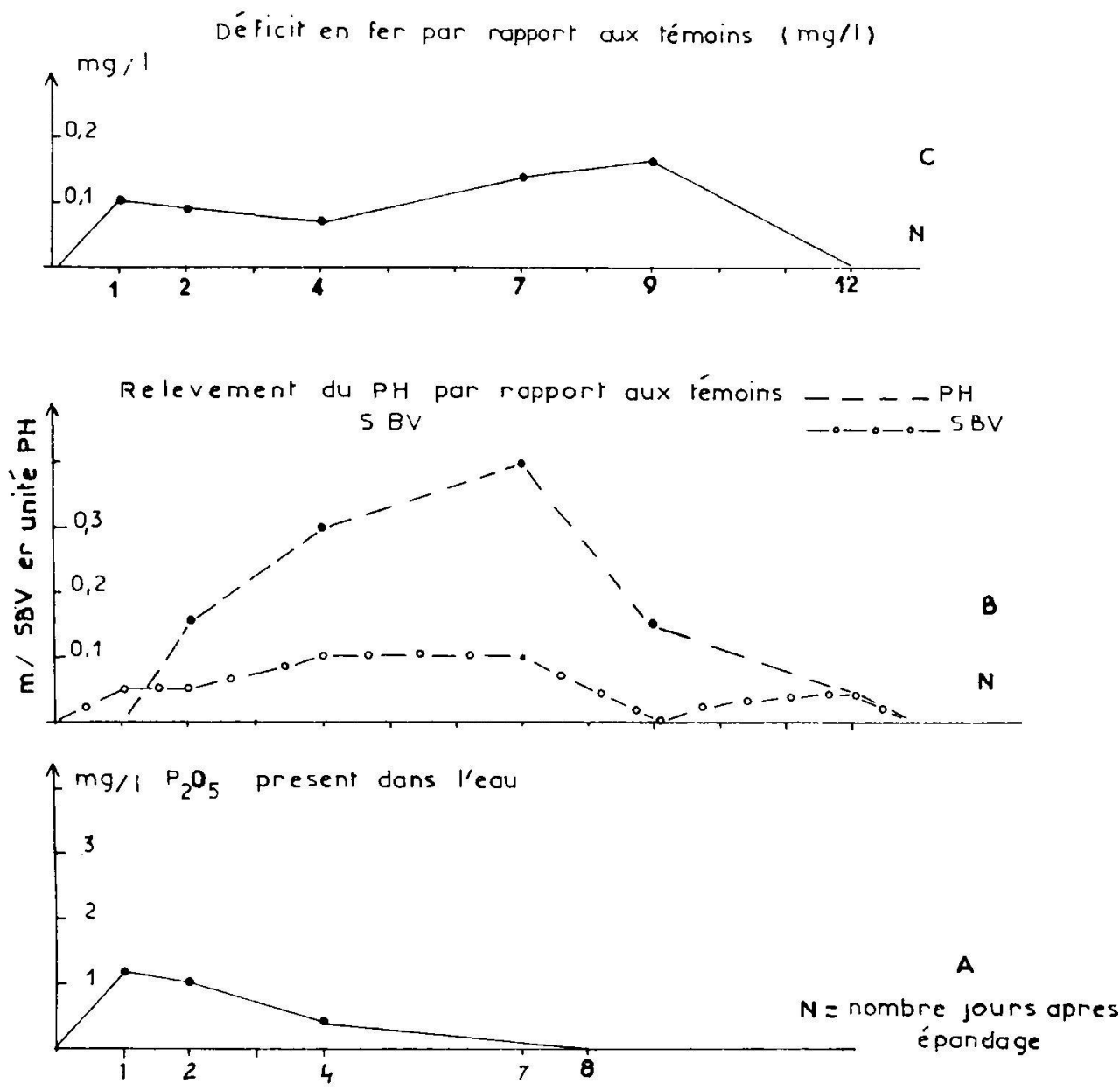
Fig. $2-40 \mathrm{~kg}$ de superphosphate à l'ha, soit $18 \mathrm{~kg} \mathrm{P}_{2} \mathrm{O}_{\mathrm{s}}$ (chaque point représente une moyenne)

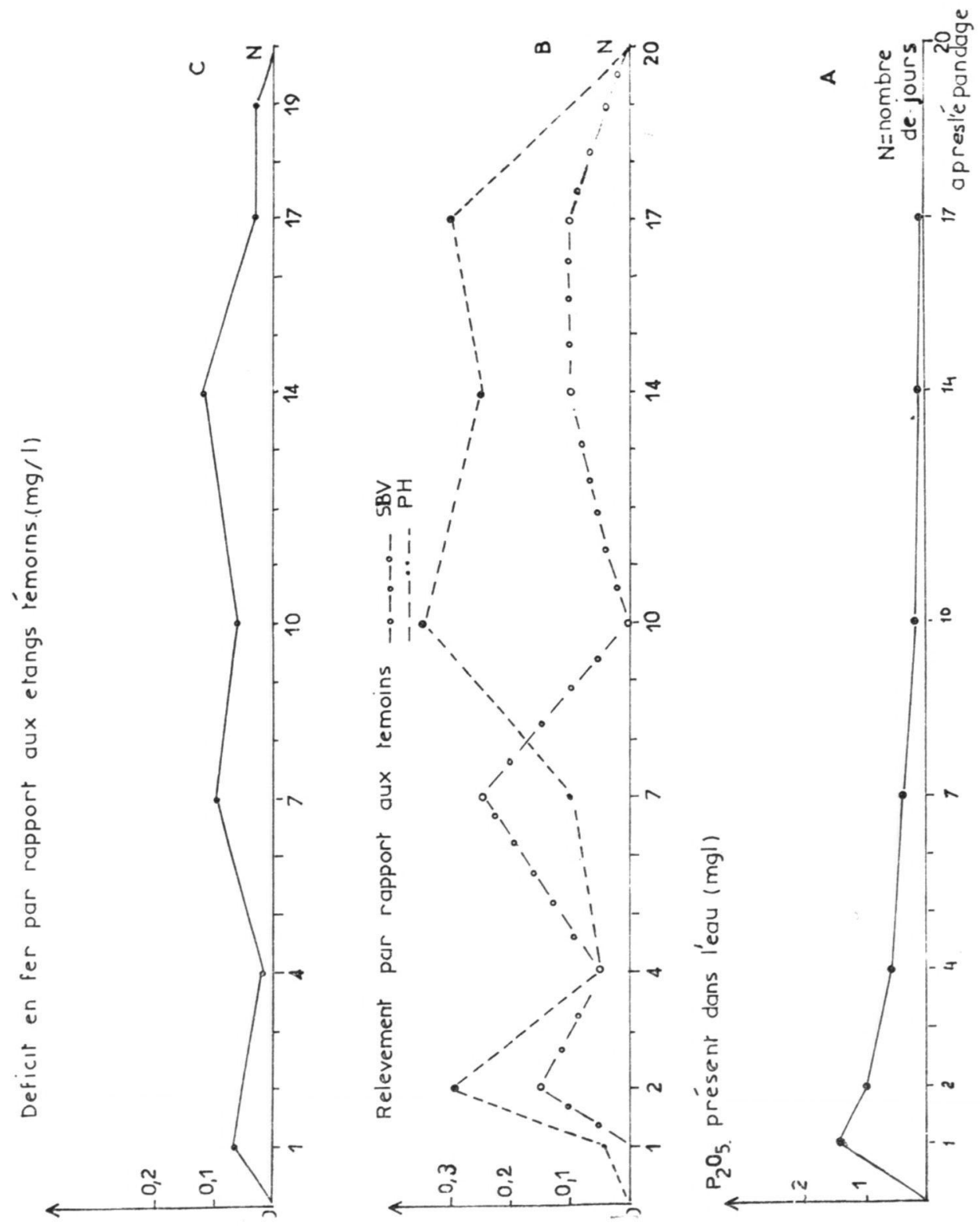


Fig. $3-80 \mathrm{~kg} / \mathrm{ha}$ de superphosphate soit $36 \mathrm{~kg} \mathrm{P}_{2} \mathrm{O}_{5}$ (chaque point représente une moyenne)
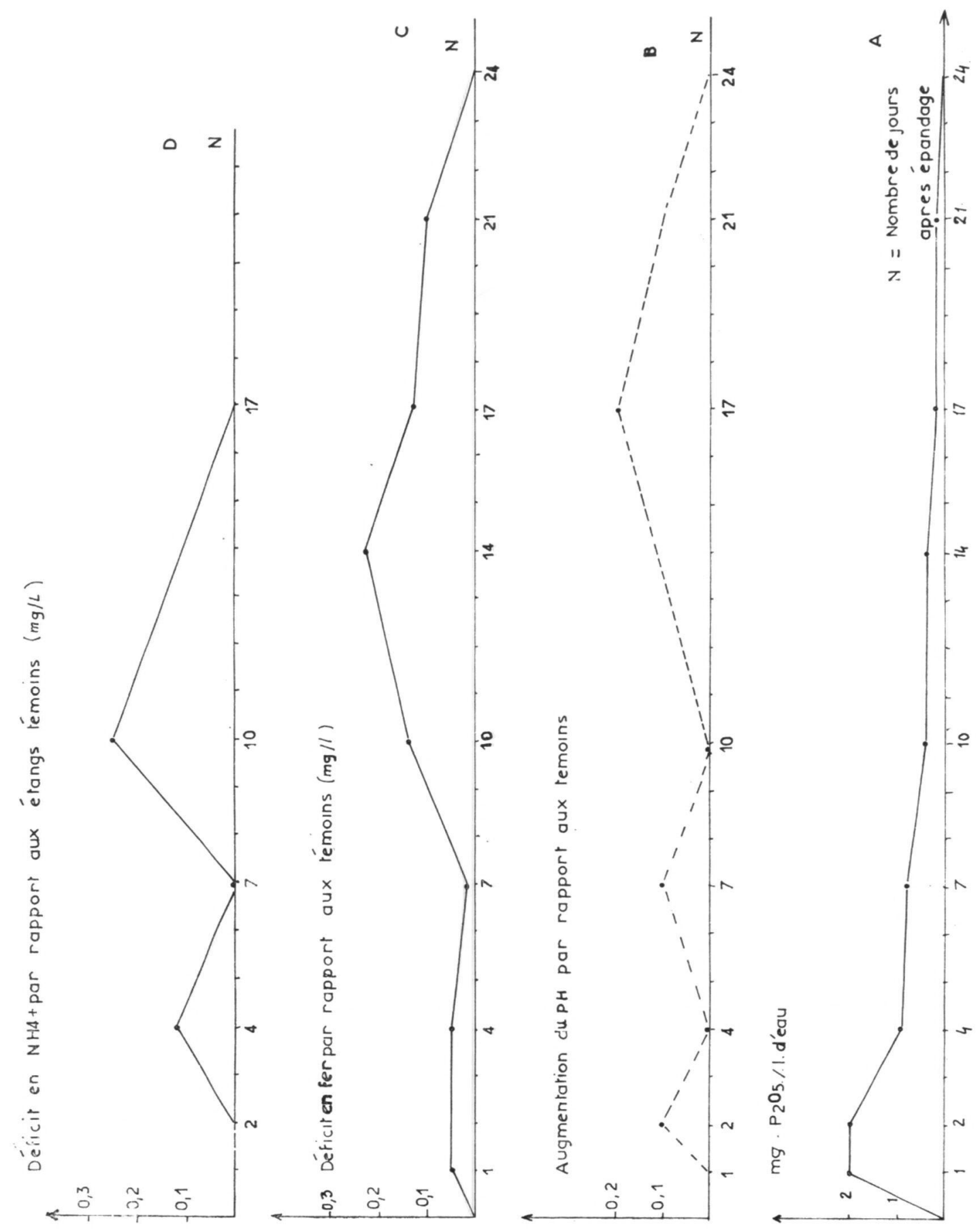
Fig. $4-120 \mathrm{~kg}$ de superphosphate ha $=54 \mathrm{~kg} \mathrm{P}_{2} \mathrm{O}_{5} / \mathrm{ha}$ (chaque point représente une moyenne)

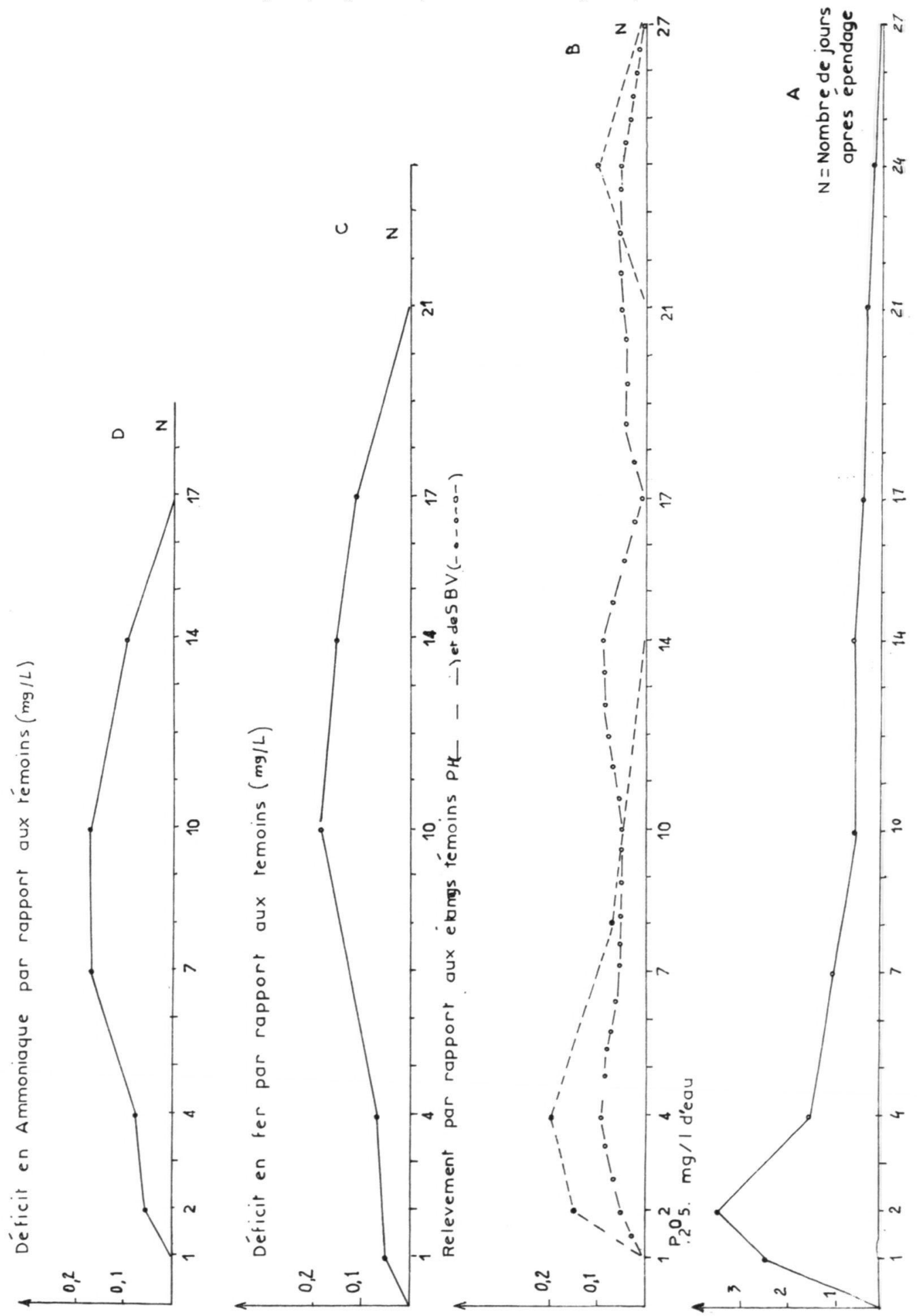


Les nitrites sont toujours présents en très petites quantités (moins de $0,015 \mathrm{mg} / \mathrm{l}$ ), et le déficit par rapport aux étangs témoins est très net, sauf pour la dose épandue la plus faible $(20 \mathrm{~kg} / \mathrm{ha})$, ainsi qu'en témoigne le tableau ci-après.

\section{TABLEAU ॥}

Déficit en nitrites

\begin{tabular}{|c|c|c|c|c|c|c|}
\hline Jour & $20 \mathrm{~kg} / \mathrm{ha}$ & $40 \mathrm{~kg} / \mathrm{ha}$ & 80 & $\mathrm{~kg} / \mathrm{ha}$ & 120 & $\mathrm{~kg} / \mathrm{ha}$ \\
\hline $\begin{array}{r}1 \\
4 \\
7 \\
10 \\
14 \\
17 \\
21\end{array}$ & $\begin{aligned} & 0,002 \\
+ & 0,001 \\
& 0,0035 \\
+ & 0,001\end{aligned}$ & $\begin{array}{l}0 \\
0,001 \\
0,0015 \\
0,0015 \\
0,004 \\
0,002\end{array}$ & + & $\begin{array}{l}0,001 \\
0 \\
0,001 \\
0,003 \\
0,003 \\
0,003 \\
0,004\end{array}$ & + & $\begin{array}{l}0,003 \\
0,001 \\
0,001 \\
0,004 \\
0,0075 \\
0,0035 \\
0,004\end{array}$ \\
\hline
\end{tabular}

N.B. : voir tableau I.

Les nitrates sont également déficitaires dans les étangs fertilisés, mais nous n'accordons qu'un crédit limité à cette observation en raison des difficultés que nous avons eu à pratiquer le dosage des nitrates.

Les mesures de résistivité et d'oxygène dissous dans l'eau ne permettent pas de tirer de conclusions nettes.

\section{DISCUSSIONS}

En ce qui concerne la seule Station de Bouakè, nos essais sont à comparer à des travaux antérieurs non publiés (LESSENT, 1970) ayant donné lieu seulement à des mesures de quantités de phosphates présents dans l'eau. Ces expériences, résumèes ci-dessous, ont donné lieu à un seul essai, sauf dans les 2 cas mentionnés.

TABLEAU III

\begin{tabular}{|c|c|c|c|c|c|c|}
\hline $\begin{array}{c}\mathrm{kg} \text { DOSES } \\
\text { phate triple/ha }\end{array}$ & 20 & 40 & 80 & 120 & 180 & 240 \\
\hline $\begin{array}{c}\text { Durée de pré- } \\
\text { sence des en- } \\
\text { grais dans l'eau }\end{array}$ & 8 jours & 18 jours & $\begin{array}{c}20 \text { jours } \\
\text { (1) }\end{array}$ & $\begin{array}{c}30 \text { jours } \\
(1)\end{array}$ & $\begin{array}{c}\text { plus de } \\
30 \text { jours } \\
\text { (2) }\end{array}$ & $\begin{array}{c}\text { plus de } \\
30 \text { jours } \\
(2)\end{array}$ \\
\hline
\end{tabular}

(1) Cette donnée est la moyenne des résultats de 2 répétitions.

(2) Le travail n'a malheureusement pas été poursuivi plus longtemps. 
La similitude entre ces derniers résultats et les nôtres est certaine ; c'est pourquoi, indépendamment de toute considération d'ordre économique, il semble possible de préconiser, dans les conditions de la Station de Bouaké, des épandages mensuels de 80 à $120 \mathrm{~kg} / \mathrm{ha}$, et par mois de superphosphate triple à $45 \%$ de $\mathrm{P}_{2} \mathrm{O}_{5}$. On remarquera, en outre, qu'il est inutile d'épandre plus de $180 \mathrm{~kg} / \mathrm{ha}$; en effet, la durée de présence des engrais dans l'eau n'est pas modifiée; la quantité d'engrais présente dans l'eau à chaque période non plus (figure 5).

Il est intéressant de comparer nos travaux à ceux d'autres auteurs ayant poursuivi des recherches analogues et, essentiellement, à ceux de M. VINCKE (1969-70) auxquels nous avons collaboré à leurs débuts. Ces travaux ont été poursuivis à la Station piscicole d'Ampamaherana, située à $1100 \mathrm{~m}$ d'altitude, en zone forestière près de Fianarantsoa, sur sol latéritique; son climat est caractérisé par une saison fraiche marquée; ils ont donné lieu à des épandages de superphosphate triple réalisés à la volée dans des étangs non empoissonnés et aux mêmes analyses d'eau que les nôtres, sauf le dosage de l'oxygène et des nitrites dissous; les doses de superphosphate triple étudiées ont été de $60,120,200$ et $300 \mathrm{~kg} / \mathrm{ha}$; un essai a été réalisé en saison chaude sur des étangs empoissonnés avec 25 carpillons à l'are.

Le premier résultat important à MADAGASCAR est l'influence des saisons qui n'a pas été observée à BOUAKE; les engrais restent moins longtemps dans l'eau en saison froide qu'en saison chaude; ceci est vérifié pour toutes les doses utilisées (fig. 6) et le rapport entre les 2 durées observées est de 1 à 3 pour la dose la plus faible $(60 \mathrm{~kg} / \mathrm{ha})$.

Les épandages successifs d'engrais dans les mêmes étangs ont été insuffisamment espacés pour permettre la détermination exacte du temps de présence des engrais aux doses élevées (de 120 à $300 \mathrm{~kg} / \mathrm{ha}$ ); mais, en hiver, le temps de présence de phosphate dans l'eau est le même pour les doses de 200 et de $300 \mathrm{~kg} / \mathrm{ha}$ et, en été, à partir du $15 \mathrm{e}$ jour, les graphiques traduisant l'évolution de la présence de phosphate dans l'eau au cours du temps se ressemblent pour les doses précitées; l'observation faite à BOUAKE, rapportée plus haut, montrant que des épandages de plus de $180 \mathrm{~kg} / \mathrm{ha}$ sont superflus, se trouve donc en partie vérifiée.

Les résultats obtenus aux doses de 60 et $120 \mathrm{~kg} / \mathrm{ha}$, comparés à ceux de nos essais de BOUAKE, révèlent en hiver un temps de présence des engrais voisin de celui que nous avons observè à BOUAKE et, en été, une durée beaucoup plus longue; ce qui inciterait à penser qu'à MADAGASCAR, l'épandage mensuel de $60 \mathrm{~kg}$ de superphosphate triple peut être préconisé sur l'ensemble de l'année. Les raisons de cette différence entre les travaux de BOUAKE et ceux de MADAGASCAR ne nous apparaissent pas pour le moment.

A la dose de $8 \mathrm{~kg} / \mathrm{ha}$ de $\mathrm{P}_{2} \mathrm{O}_{5}$, soit $18 \mathrm{~kg} / \mathrm{ha}$ de superphosphate triple, RABANAL (1968), sur des étangs empoissonnés avec des carpes, note une durée de présence de l'engrais de 10 jours, ce qui est un peu plus long que celle observée par nous avec $20 \mathrm{~kg} / \mathrm{ha}$.

II émet l'hypothèse selon laquelle les carpes, en fouillant dans le sol, relibèrent le phosphate dans les conditions dans !esquelles il travaille (en ALABAMA aux USA). Nous avons tenté de faire piétiner les étangs après la disparition des phosphates pour voir si les engrais ne seraient pas libérés 
Fig. 5 - Evolution de la quantité d'engrais présent dans l'eau après épendage de quantités élevées d'engrais (Station de Bouake)

- . . . . . . $180 \mathrm{~kg} / \mathrm{ha}$

. . . . . . . $240 \mathrm{~kg} / \mathrm{ha}$

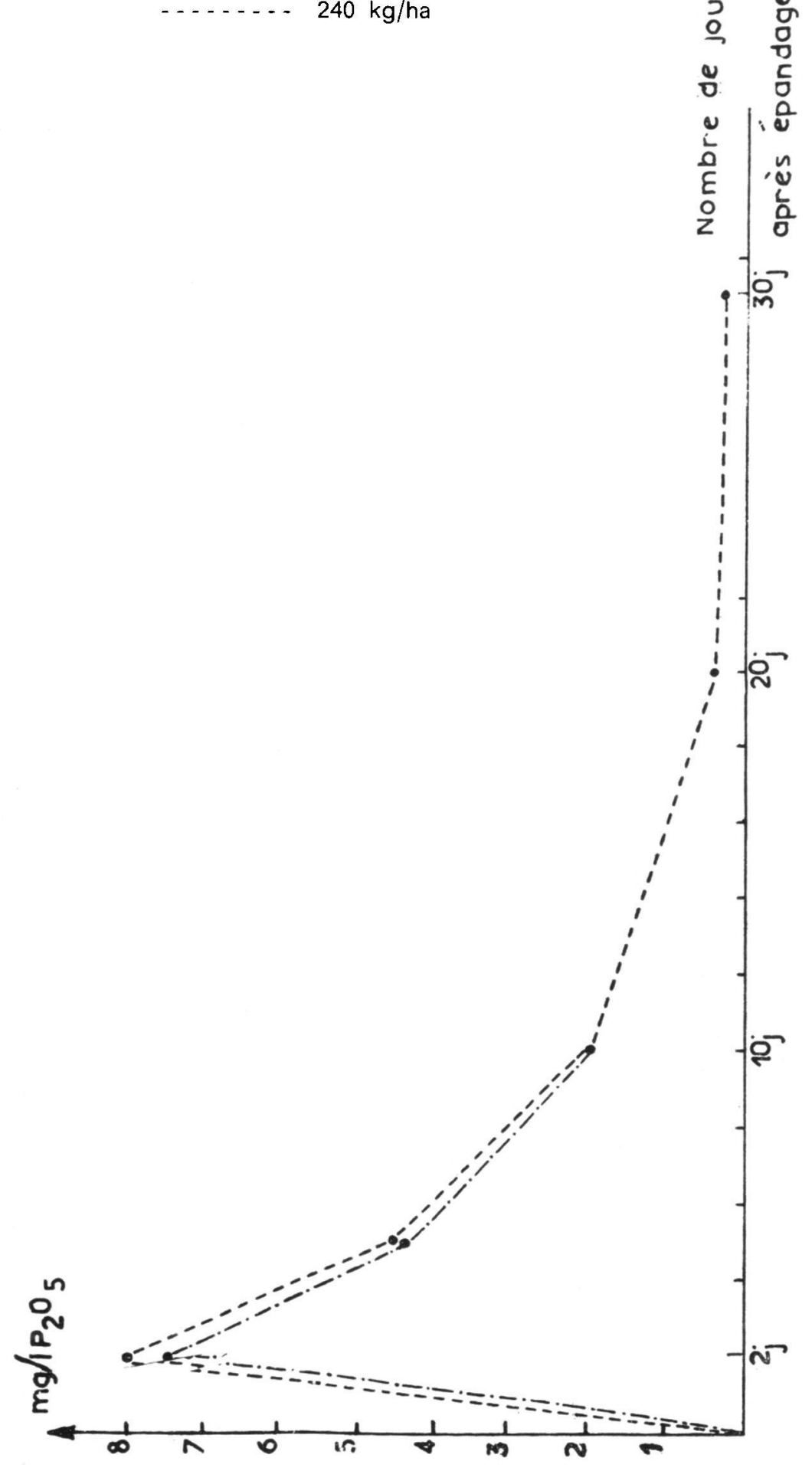


Fig. 6 - Durée de présence des engrais phosphatés dans l'eau (d'après VINCKE à Madagascar)

-. - . Saison froide Saison chaude

(8), (6), (4) Nombre o'e mesures (s'il est supérieur à 3)
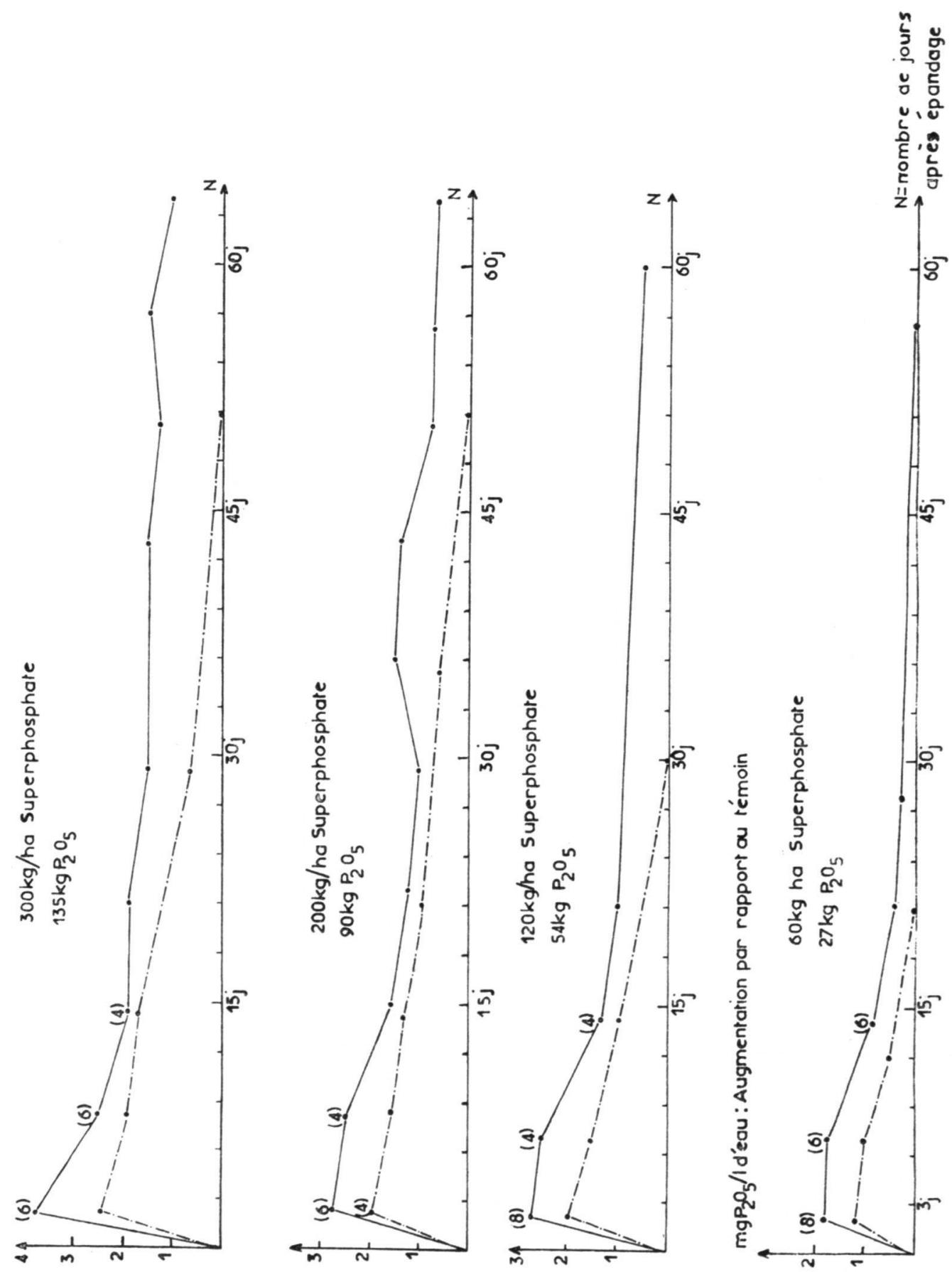
de cette façon; sauf dans $5 \%$ des cas, il semble qu'il n'en ait rien été. La présence des poissons, que ce soit les Tilapia hybrides monosexes à BOUAKE ou les carpillons à MADAGASCAR, n'a pas eu non plus d'influence appréciable.

Parmi les variations des conditions de milieu notées, par rapport aux étangs tèmoins, la plus intéressante nous parait ètre le déficit général en fer des étangs fertilisés, déficit qui dure plus ou moins longtemps suivant les doses ; cette observation n'a pas èté faite à MADAGASCAR, car la technique de mesure de la teneur en fer ne permettait pas de déceler les faibles doses. Une telle observation révèle d'abord une amélioration de l'état sanitaire de l'étang liée à la fertilisation phosphatée; elle est importante, car à de très faibles doses $(0,9 \mathrm{mg} / \mathrm{l})$, le fer est toxique pour les poissons dans des eaux de $\mathrm{pH}$ compris entre 6,5 et 7,5 d'après BANDT, rapporté par SCHAPERCLAUS; or, les eaux de BOUAKE avaient souvent un $\mathrm{pH}$ compris entre 7 et 7,5 .

Plus généralement, ce déficit en fer des étangs fertilisés confirme, à la suite de SCHAPERCLAUS (1962) et GOLTERMAN (1968), qu'il existe dans les eaux de BOUAKE une relation entre le métabolisme des phosphates et celui du fer.

Cette observation, jointe à ce qui a été dit plus haut (absence d'effet du piétinement des étangs), tend à prouver qu'une partie au moins des phosphates se trouve précipitée de façon irréversible sous forme de $\mathrm{Fe}$ (PO4) 3.

Une autre partie des phosphates est sans doute absorbée par $\mathrm{Fe}(\mathrm{OH}) 3$ qui précipite vraisemblablement dans les conditions d'alcalinité de nos étangs; d'après SCHAPERCLAUS, la proportion de phosphate absorbé de cette façon varie avec le $\mathrm{pH}$, mais elle n'est pas appréciable quantitativement dans le cas présent.

De toute façon, s'il en est ainsi, cette dernière partie des phosphates est relibérable par la vase dans l'eau (GOLTERMAN), puisque ces phénomènes ont lieu sur une très petite épaisseur de vase (moins de $1 \mathrm{~mm}$ ). Dans notre cas, ces échanges s'ils ont eu lieu se sont passés avant que les phosphates dissous aient totalement disparu; sinon, on aurait retrouvé systematiquement dans tous les étangs les phosphates dissous après piétinement. Quelquefois la présence de poissons fouisseurs permet la libération totale de phosphates absorbés par $\mathrm{Fe}(\mathrm{OH}) 3$, ce qui expliquerait l'observation de RABANAL (1968), évoquée plus haut.

Ces éléments de discussion n'imposent pas l'absence d'autres mécanismes de mobilisation des phosphates, notamment avec le calcium; mais, si ces mécanismes existent, nous n'avions pas les moyens de les mettre en évidence.

Le relèvement du PH et du SBV, constaté dans nos étangs, n'a pas été observé à Madagascar, malgré l'acidité des eaux locales; cela aurait été du plus haut intérêt, puisque les eaux se seraient trouvées rapprochées de la neutralité que l'on sait être en général le milieu optimum pour les poissons. A Bouaké, un chaulage supplémentaire est inutile ; il aurait un effet marqué à une dose élevée devenant prohibitif financièrement (LESSENT, 1968).

La dernière remarque intéressante concerne l'abaissement des taux d'Ammoniaque, lorsque les doses de phosphates sont très élevées. Cette observation, comme celle qui a été faite au sujet du fer (voir ci-dessus), corrobore l'affirmation de HUET (1970), selon laquelle la fertilisation améliore l'état sanitaire des étangs. De toute façon, l'Ammoniaque est présente dans le milieu à des doses non nocives pour les poissons; ce problème doit être abordé dans l'étude de la fumure azotée. 


\section{CONCLUSION}

Les résultats essentiels de ces travaux sont la disparition assez rapide de l'effet des engrais phosphatés dans les étangs et les conséquences à en tirer pour la pratique de la fertilisation. Si on adopte un rythme d'épandage mensuel, ce qui parait être la norme, les doses de $80 \mathrm{~kg} / \mathrm{ha}$ et de $120 \mathrm{~kg} / \mathrm{ha}$ seront à retenir; si la dose de $120 \mathrm{~kg} / \mathrm{ha}$ s'avère prohibitive financièrement, on adoptera celle de $80 \mathrm{~kg} / \mathrm{ha}$ de superphosphate triple à $45 \%$ de $P_{2} \mathrm{O}_{5}$. Une partie de l'engrais est certainement absorbée directement par le phytoplancton; le restant est, soit précipité directement sous forme de $\mathrm{Fe}(\mathrm{PO} 4) 3$, soit absorbé par $\mathrm{Fe}(\mathrm{OH}) 3$, auquel cas, il peut être relibéré. Appliquée à des doses élevées, la fumure phosphatée améliore l'état sanitaire de l'étang et provoque un relèvement sensible du $\mathrm{PH}$.

\section{Remerciements}

L'auteur remercie M. VINCKE qui lui a communiqué certains résultats de ses recherches personnelles sur la fertilisation phosphatée à Madagascar.

\section{BIBLIOGRAPHIE}

C.T.F.T., 1970 - Rapport annuel sur les Pèches continentales au Centre Technique Forestier Tropical - Ed. C.T.F.T. Nogent/s/Marne - 70 pages.

F.A.O., 1968 - Actes du symposium mondial sur la pisciculture en étang à température élevée. Fertilisation et rôle du sol dans la productivité. F.A.O., Fish. Rep. 44 (3) p. 1 à 274.

GOLTERMAN H L, 1968 - Influence of soil on the chemistry of water in relation to productivity. F.A.O. Fish. Rep. 44 (3) p. 168 à 182.

HUET, 1970 - Traité de Pisciculture - Ed. Ch. Wyngaert, Bruxelles - 600 p.

LESSENT P., 1968 - Essai de fertilisation des étangs à la Station Piscicole de Bouakè - F.A.O. Fish. Rep. 44 (3) p. 95 à 100.

RABANAL R. R., 1968 - Inorganic fertilizers for pond fish culture - F.A.O. Fish Rep. 44 (3) p. 164 à 167.

SCHAPERCLAUS, 1962 - Traité de Pisciculture en étang - Vigot Frères - Editeurs Paris.

VINCKE M. 1969 - Recherches sur la fertilisation des étangs. Compte rendu d'activité - C.T.F.T. Madagascar, p. 27 à 34 - Document dactylographié à diffusion restreinte. 